\title{
Design of Integrated Limited-Stop and Short-Turn Services for a Bus Route
}

\author{
Hu Zhang, Shuzhi Zhao, Huasheng Liu, and Shidong Liang \\ College of Transportation, Jilin University, Changchun 130022, China \\ Correspondence should be addressed to Huasheng Liu; liuhuasheng521@163.com
}

Received 18 February 2016; Revised 22 June 2016; Accepted 3 July 2016

Academic Editor: Haipeng Peng

Copyright (C) 2016 Hu Zhang et al. This is an open access article distributed under the Creative Commons Attribution License, which permits unrestricted use, distribution, and reproduction in any medium, provided the original work is properly cited.

As a major choice for daily travel, public transit plays an important role in transporting passengers, thus relieving congestion on urban transit routes. In high-demand bus networks, urban transit demand presents imbalance of use of urban bus corridors. The demand patterns in both directions are asymmetric. In this paper, we develop a model which calculates the network and transportation costs in terms of wait time, in-vehicle travel time, and operator costs. We propose an integrated strategy, with an integrated limited-stop and short-turn line, by adjusting a variety of frequencies to meet the unbalanced and asymmetric demand. To minimize these costs, a model with a genetic algorithm can determine frequencies and the proper stations which can be skipped, as well as where turning back can occur, given an origin-destination trip matrix. Numerical examples are optimized to test the availability of an integrated service by minimizing the objective function, and the results are analyzed. Our results show that integrated service patterns can be adjusted to meet the demand under different conditions. In addition, the optimized schemes of an integrated service and the frequencies derived from the model can significantly reduce total cost.

\section{Introduction}

In an urban transit system, demand presents different patterns in high-demand corridors. In such areas, a subset of stations which experience high demand should be served by providing greater bus supply. In addition, those stations with high demand are different in two directions along the entire route. If all stations are given a high level of service, offering more benefits to users than operators would make the transit system inefficient, due to increased operator costs at the low demand stations. Thus, an optimal design of bus services is needed to meet the demand in the different patterns. The design should provide different schemes to the stations with high and low demand. The frequency of service should be increased at stations with high demand, while stations with low demand could be skipped by some buses in order to shorten travel times. In addition, the transit systems would operate more efficiently, because they would consider both user and operator costs.

To provide an optimal frequency of service at different stations and in order to solve the problems of unbalance and asymmetric demand patterns, a number of operational strategies have been proposed [1-5]. A short-turn service allows some buses to serve shorter cycles when operating on those sections of high demand in both directions. Deadheading would allow empty vehicles to skip stations in the lowdemand direction and to mainly serve stations in the highdemand direction. Limited-stop services only serve stations with high demand in order to shorten travel times.

In their study on short turning, Jordan and Turnquist [6] implemented zone scheduling to improve service reliability on a bus route which they divided into specific zones. Furth [7] and Ceder [8] determined the frequency of vehicles on short-turn lines, which was equivalent to the frequency of vehicles serving all the stations, multiplied by the parameter $n$. Delle Site and Filippi [9] determined the stations which should be served by vehicles operating on the short-turn lines and the optimum frequency of vehicles for short-turn and all-stop services, by minimizing the sum of user costs and operator costs.

With regard to deadheading, Furth [10] proposed alternating deadheading for urban bus routes. Considering a reduction in passenger waiting times, the number of vehicles was calculated under the usual scheduling constraints. 
Eberlein et al. [11] used deadheading as a real-time control strategy to improve the efficiency of transit systems. Liu et al. [12] would allow the bus to skip one or more stops to reduce travel time when a bus was late and behind schedule. Using Genetic Algorithm Incorporating Monte Carlo Simulation, the optimization model was solved.

Various works in existing literature focus on limitedstop services. Afanasiev and Liberman [13] categorized bus services as normal, limited-stop, and express. The authors also addressed the design of limited-stop lines using passenger demand to determine the beginning and end of each limited-stop line. Silverman [14] adopted a limitedstop service strategy to improve bus speed and reliability, based on adjusting time spans and scheduling. Tétreault and El-Geneidy [15], using AVL and APC data, estimated the running time for a limited-stop line in parallel with a normal line. Considering capacity constraints, Leiva et al. [16] proposed a method with vehicle capacity constraints and transfers to determine the optimum frequencies, vehicle types, and serving stations. Feng et al. [17] analyzed the travel time model under mixed traffic conditions. Feng et al. proposed an optimization model for designing skip-stop services that could minimize the total travel time for both car travelers and bus travelers. Based on a cost analysis of the Beijing BRT Line 2, Chen et al. [18] integrated optimization of that service's headways and stop-skipping strategy. The results showed that when the optimum headway is between 3.5 and $5.5 \mathrm{~min}$ for skip-stopping services, the user costs can be greatly reduced. Given predetermined train skipstop patterns, Niu et al. [19] proposed a unified quadratic integer programming model with linear constraints. Niu et al's model minimized the total passenger waiting time at stations by computing and adjusting the train timetables for a rail corridor. Chen et al. [20] proposed that all vehicles should be allowed to skip stations, but no station could be skipped by two consecutive vehicles, taking into consideration vehicle capacity and stochastic travel times.

Meanwhile, integrated strategies have been applied to the optimization of transit services. Cortés et al. [21] developed a model which implemented integrated deadheading and short-turn strategy for a single line. The model determined the frequencies within serving zones that provided an integrated strategy or no strategy, as well as the stations where the strategy would begin and end.

The previous strategies are implemented to improve the service level of the transit systems, and integrated strategies can be applied to more demand patterns. However, the integration of limited-stop and short-turn strategies is promising and exposes the means to achieve optimization of transit services. For some stations with high demand, a limited-stop service could be effective. For other stations, a short-turn service might be better. Therefore, the integration of limited-stop and short-turn strategies should be useful, and the performance of such integrated strategies is worth analyzing. Following this introduction, Section 2 analyzes the characterization of demand and defines the integrated strategy in order to estimate demand when selecting and implementing the integrated strategy. Section 3 formulates the model that minimizes the total costs for pure limited-stop

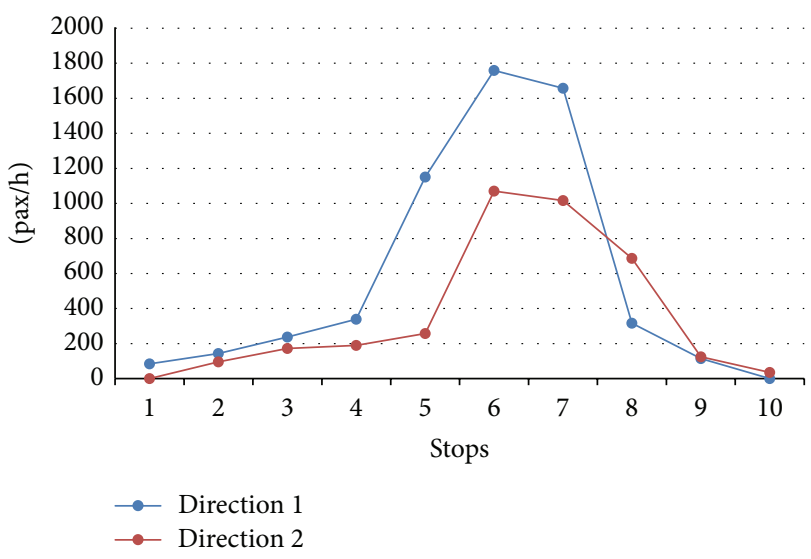

FIGURE 1: Load profile of a corridor with spatial concentration of the demand.

services and the integration of limited-stop and short-turn services. Section 4 describes the solution methods for our proposed model. Section 5 analyzes the results of our numerical examples. Finally, Section 6 presents our conclusions.

\section{Problem Description}

Figures 1 and 2 show a typical load profile on a transit corridor, as well as the passenger boarding and alighting rates at 10 stations in Changchun, China, during a peak morning hour, in both direction 1 and direction 2 . In direction 1 , the load is concentrated between station 5 and station 7. The boarding or alighting demand at stations 5,6 , and 8 is high. In direction 2, the load is concentrated between station 6 and station 8 , and the stations with the highest boarding or alighting demands are stations 5, 7, and 8. In this particular case, a traditional service, whereby all buses serve every station, is not the most efficient choice, because the stations with low demand (such as stations 1, 2, 3, 4, 7, and 10) in direction 1 might not need to be serviced by every bus. We provide a suitable integrated strategy, whereby a fleet of buses (hereinafter referred to as fleet A) serves all stations. The other buses (hereinafter referred to as fleet B) only serve those stations with high demand. When fleet B operates in direction 1, skipping stations from 1 to 4 , these buses run without passengers in order to provide a shorter running time. In addition, by skipping station 7 , fleet B buses run with passengers for a shorter in-vehicle time by providing only limited stops. After serving station 9, fleet B buses turn back and start serving passengers in direction 2 but only until station 4 . Fleet B buses then turn back again, thus creating a shorter (or short-turning) cycle.

Figure 3 shows the operational scheme of integrated limited-stop and short-turn strategies. The scheme comprises all-stop, limited-stop, and short-turn services. Fleet A provides an all-stop service, serving every station, and the frequency of fleet $\mathrm{A}$ is denoted as $f_{\mathrm{A}}$. Fleet $\mathrm{B}$ provides a limited-stop service, serving stations with high demand. The frequency of fleet $\mathrm{B}$ is denoted as $f_{\mathrm{B}}$. When fleet $\mathrm{B}$ runs without passengers in both directions at either end of 


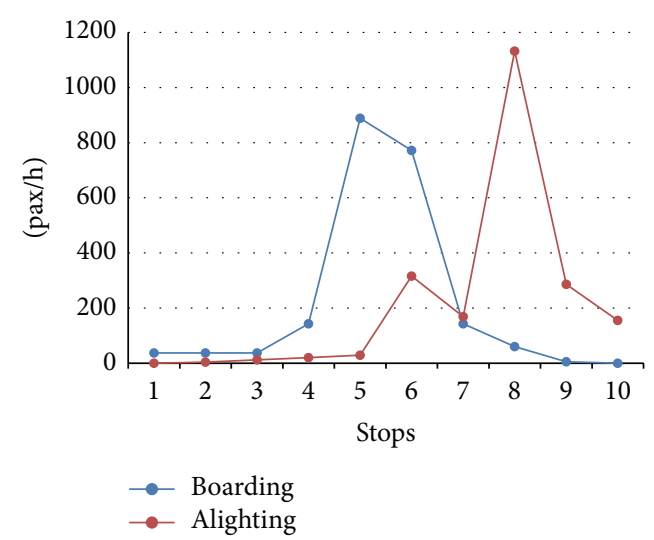

(a)

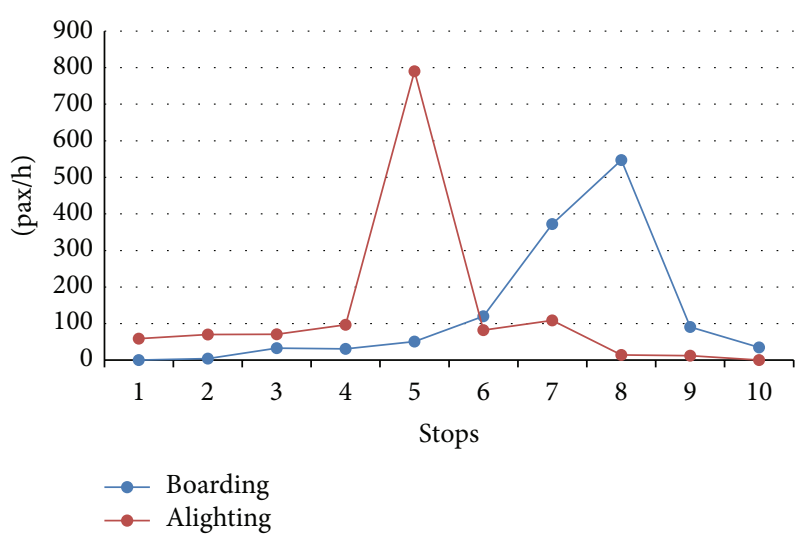

(b)

FIGURE 2: Passenger boarding rate and alighting rate: (a) direction 1; (b) direction 2 .

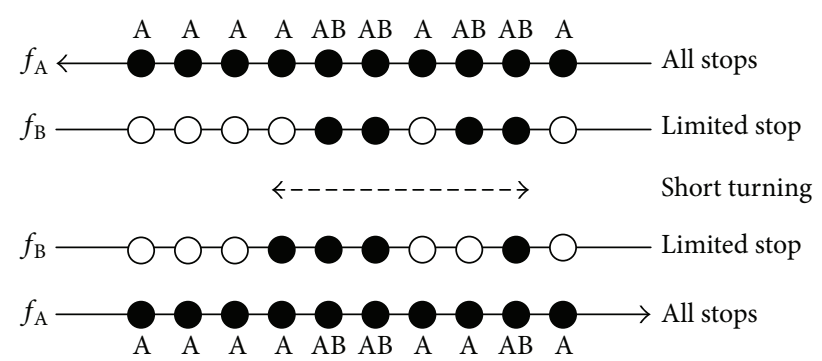

Figure 3: Operational scheme of integrated limited-stop and shortturning strategies.

the line and there are appropriate turning points, a shortturn service will be provided. The stations can be defined as two types as follows: A stations are only served by fleet $A$, while $A B$ stations are served by both fleet $A$ and fleet B. To implement the integrated strategy, the first task is to select the suitable stations for the limited-stop service to skip. If there are no skipped stations to shorten the running time or in-vehicle time for fleet B, then the limited-stop and short-turn services will disappear. All buses will serve all stations. If there are no stations where buses run without passengers, then a short-turn service cannot be implemented, and the integrated strategy would just comprise all-stop and limited-stop services. Therefore, we need a model and solution method which provides for an integrated strategy. The key function of that model, method, and strategy will be to determine which stations should be skipped, as well as the optimized frequencies for both fleet A and fleet B.

Under an integrated strategy, passengers can select fleet A or fleet B at any of the stations served by both all-stop and limited-stop buses. When the starting or ending stations are A stations (therefore only served by fleet A), passengers will endure stops at all stations along their route. When the starting and ending stations are $\mathrm{AB}$ stations, passengers can then select fleet A or fleet B. If they select fleet B, passengers may still have to stop at all stations (if there are no skipped stations on their route), which is similar to selecting fleet A. Alternatively, their travel time will be reduced if there are skipped stations on the route. Thus, by considering the types of stations which passengers travel via, we can classify customer demand into three categories, denoted as $\mathrm{OD}_{i j 1}$, $\mathrm{OD}_{i j 2}$, and $\mathrm{OD}_{i j 3}$, in Table 1. The demand from station $i$ to station $j$ can be denoted as $\lambda_{i j}$. For the first type of demand, the origin or destination of passengers is an A station. In this case, passengers can only select fleet $\mathrm{A}$. For the second and third types of demand, both the origin and destination of passengers are $A B$ stations. In these cases, passengers can select fleet $\mathrm{A}$ and fleet $\mathrm{B}$, while the second demand-type passengers (riding fleet B) will stop at each station, as there are no skipped stations. The third demand-type passengers need to skip some stations.

Then the demand served by fleet $\mathrm{A}$ is following as $\mathrm{OD}_{i j 1}+$ $\left(f_{\mathrm{A}} /\left(f_{\mathrm{A}}+f_{\mathrm{B}}\right)\right) \mathrm{OD}_{i j 2}+\left(f_{\mathrm{A}} /\left(f_{\mathrm{A}}+f_{\mathrm{B}}\right)\right) \mathrm{OD}_{i j 3}$, while the demand served by fleet $\mathrm{B}$ is following as $\left(f_{\mathrm{B}} /\left(f_{\mathrm{A}}+f_{\mathrm{B}}\right)\right) \mathrm{OD}_{i j 2}+$ $\left(f_{\mathrm{B}} /\left(f_{\mathrm{A}}+f_{\mathrm{B}}\right)\right) \mathrm{OD}_{i j 3}$.

\section{Design Model of Limited Stop and Short Turning}

On the basis of the aforementioned observations, a short-turn service is based on a limited-stop service. Without limited stops, the timing for turning back will disappear. Thus, we first determine the design model of the limited-stop service, and we then select the timing of short turning. As the model should reflect benefits for both users (passengers) and the operator (buses), the formulated models are cost functions of the transit system. The costs of the transit system include user costs and operator costs. Here, user costs are associated with waiting time and in-vehicle time, while operator costs relate to the bus running times. To minimize the cost functions (including user and operator costs), an integrated strategy scheme can be determined, and the frequencies of fleet $\mathrm{A}$ and fleet $B$ can be adjusted. Thus, the remainder of this section of our study contains a design model of a limited-stop line without short turning; we then present a model for a limitedstop and a short-turn line.

3.1. Model for Limited-Stop Line without Short Turning. For a limited-stop line without short turning, passengers from the different demand categories (such as $\mathrm{OD}_{i j 1}, \mathrm{OD}_{i j 2}$, and $\mathrm{OD}_{i j 3}$ ) may choose fleet A or fleet $\mathrm{B}$. These choices, in turn, cause 
TABLE 1: Classification of demand.

\begin{tabular}{lcc}
\hline Categories of the demand & Demand of categories & Configuration of categories \\
$\mathrm{A}-\mathrm{A}, \mathrm{A}-\mathrm{AB}, \mathrm{AB}-\mathrm{A}$ & $\mathrm{OD}_{i j 1}=\lambda_{i j} \cdot \max \left[\left(1-x_{i}\right),\left(1-x_{j}\right)\right], \quad \forall i, j$ & $\mathrm{OD}_{i j 2}=\lambda_{i j} \cdot x_{i} x_{j}$ for $j=i+1$ \\
$\mathrm{AB}-\mathrm{AB}$ & $\mathrm{OD}_{i j 3}=\lambda_{i j} \cdot x_{i}\left(1-x_{j}\right) x_{k}$ for $i<j<k$ & $\mathrm{AB}$ \\
$\mathrm{AB}-\mathrm{A}-\mathrm{AB}$ & & $\mathrm{AB}$
\end{tabular}

Variable $x_{i}$ indicates the type of station $i$. Thus, $x_{i}=1$, and station $i$ is AB station; $x_{i}=0$, and station $i$ is A station, $\forall i$.

a variety of waiting times and in-vehicle times. We assume that passengers arrive at stations at a fixed rate and that buses arrive at regularly spaced intervals. These assumptions are suitable in high-demand scenarios. The average passenger waiting time is half of the headway. Headway, for A stations, is equal to $1 / f_{\mathrm{A}}$. For $\mathrm{AB}$ stations, headway is equal to $1 /\left(f_{\mathrm{A}}+\right.$ $f_{\mathrm{B}}$ ). Thus, the waiting time cost at $\mathrm{A}$ stations with different demand in one direction, namely, $C_{W}^{\mathrm{A}}$, can be expressed by

$$
\begin{aligned}
C_{W}^{\mathrm{A}} & =W \cdot\left(\frac{1}{2}\right) \cdot\left[\left(\frac{1}{f_{\mathrm{A}}}\right) \cdot \sum_{i=1}^{N} \sum_{j=i+1}^{N} \mathrm{OD}_{i j 1}+\frac{1}{f_{\mathrm{A}}+f_{\mathrm{B}}}\right. \\
& \cdot\left(\sum_{i=1}^{N} \sum_{j=i+1}^{N} \frac{f_{\mathrm{A}}}{f_{\mathrm{A}}+f_{\mathrm{B}}} \mathrm{OD}_{i j 2}\right. \\
& \left.\left.+\sum_{i=1}^{N} \sum_{j=i+1}^{N} \frac{f_{\mathrm{A}}}{f_{\mathrm{A}}+f_{\mathrm{B}}} \mathrm{OD}_{i j 3}\right)\right],
\end{aligned}
$$

where $W$ is the waiting time value and $N$ is number of stations in a bus line.

Similarly, the waiting time cost at $\mathrm{AB}$ stations with different demand in one direction (named $C_{W}^{\mathrm{B}}$ ) can be expressed by

$$
\begin{aligned}
C_{W}^{\mathrm{B}} & =W \cdot\left(\frac{1}{2}\right) \cdot\left[\frac { 1 } { f _ { \mathrm { A } } + f _ { \mathrm { B } } } \cdot \left(\sum_{i=1}^{N} \sum_{j=i+1}^{N} \frac{f_{\mathrm{B}}}{f_{\mathrm{A}}+f_{\mathrm{B}}} \mathrm{OD}_{i j 2}\right.\right. \\
& \left.\left.+\sum_{i=1}^{N} \sum_{j=i+1}^{N} \frac{f_{\mathrm{B}}}{f_{\mathrm{A}}+f_{\mathrm{B}}} \mathrm{OD}_{i j 3}\right)\right] .
\end{aligned}
$$

The total waiting time cost at all stations in one direction, $C_{W}$, is sum of $C_{W}^{\mathrm{A}}+C_{W}^{\mathrm{B}}$.

As regards in-vehicle travel time costs, the in-vehicle time includes station delays (such as dwelling time and acceleration or deceleration delays) and the running time between stations. The in-vehicle time of those passengers who select fleet B may be shorter than that of those who select fleet A as a result of potentially skipped stations. The acceleration or deceleration delay is equal to $v / g$, where $v$ is the bus speed and $g$ is the bus accelerated speed. Dwell time at stations during passengers' trips depends on the boarding and alighting processes. The passenger boarding rate at all stations is the sum of $\mathrm{O} / \mathrm{D}$ matrix row vectors, while the passenger alighting rate at stations is the sum of $\mathrm{O} / \mathrm{D}$ matrix column vectors. Thus,

$$
\begin{aligned}
\lambda_{k}^{\mathrm{A}+} & =\sum_{j=k+1}^{N}\left(\mathrm{OD}_{k j 1} \cdot \frac{1}{f_{\mathrm{A}}}+\mathrm{OD}_{k j 2} \cdot \frac{1}{f_{\mathrm{A}}+f_{\mathrm{B}}}+\mathrm{OD}_{k j 3}\right. \\
& \left.\cdot \frac{1}{f_{\mathrm{A}}+f_{\mathrm{B}}}\right), \\
\lambda_{k}^{\mathrm{A}-} & =\sum_{i=1}^{k-1}\left(\mathrm{OD}_{i k 1} \cdot \frac{1}{f_{\mathrm{A}}}+\mathrm{OD}_{i k 2} \cdot \frac{1}{f_{\mathrm{A}}+f_{\mathrm{B}}}+\mathrm{OD}_{k j 3}\right. \\
\cdot & \left.\frac{1}{f_{\mathrm{A}}+f_{\mathrm{B}}}\right), \\
\lambda_{k}^{\mathrm{B}+} & =\sum_{j=k+1}^{N}\left(\mathrm{OD}_{k j 2} \cdot \frac{1}{f_{\mathrm{A}}+f_{\mathrm{B}}}+\mathrm{OD}_{k j 3} \cdot \frac{1}{f_{\mathrm{A}}+f_{\mathrm{B}}}\right), \\
\lambda_{k}^{\mathrm{B}-} & =\sum_{i=1}^{k-1}\left(\mathrm{OD}_{i k 2} \cdot \frac{1}{f_{\mathrm{A}}+f_{\mathrm{B}}}+\mathrm{OD}_{i k 3} \cdot \frac{1}{f_{\mathrm{A}}+f_{\mathrm{B}}}\right),
\end{aligned}
$$

where $\lambda_{k}^{\mathrm{A}+}, \lambda_{k}^{\mathrm{A}-}, \lambda_{k}^{\mathrm{B}+}$, and $\lambda_{k}^{\mathrm{B}-}$ are, respectively, the passenger boarding rate and alighting rate at station $k$, where passengers can select fleet A and fleet B.

Therefore, the passenger in-vehicle time cost when riding fleet A with different demand in one direction, namely, $C_{v}^{\mathrm{A}}$, can be as follows:

$$
\begin{aligned}
C_{v}^{\mathrm{A}} & =V \\
& \cdot\left\{\sum_{i=1}^{N} \sum_{j=i+1}^{N}\left[\sum_{k=i}^{j-1}\left(\frac{d_{k}}{v}+\max \left[a \cdot \lambda_{k}^{\mathrm{A}+}, b \cdot \lambda_{k}^{\mathrm{A}-}\right]+\frac{v}{g}\right)\right]\right. \\
& \left.\cdot\left(\mathrm{OD}_{i j 1}+\mathrm{OD}_{i j 2} \cdot \frac{f_{\mathrm{A}}}{f_{\mathrm{A}}+f_{\mathrm{B}}}+\mathrm{OD}_{i j 3} \cdot \frac{f_{\mathrm{A}}}{f_{\mathrm{A}}+f_{\mathrm{B}}}\right)\right\}
\end{aligned}
$$

where $V$ is the in-vehicle time value; $d_{k}$ is the spacing between stations $k$ and $k+1 ; a$ and $b$ are the average passenger boarding and alighting times.

The passenger in-vehicle time cost when riding fleet $\mathrm{B}$ with different demand in one direction (named $C_{v}^{\mathrm{B}}$ ) can be as follows: 


$$
\begin{aligned}
C_{v}^{\mathrm{B}} & =V \\
& \cdot\left\{\sum_{i=1}^{N} \sum_{j=i+1}^{N}\left[\sum_{k=i}^{j-1}\left(\frac{d_{k}}{v}+\max \left[a \cdot \lambda_{k}^{\mathrm{B}+}, b \cdot \lambda_{k}^{\mathrm{B}-}\right]+\frac{v}{g}\right) \cdot \mathrm{OD}_{i j 2} \cdot \frac{f_{\mathrm{B}}}{f_{\mathrm{A}}+f_{\mathrm{B}}}+\sum_{k=i}^{j-1}\left(\frac{d_{k}}{v}+\max \left[a \cdot \lambda_{k}^{\mathrm{B}+}, b \cdot \lambda_{k}^{\mathrm{B}-}\right]+\frac{v}{g} \cdot x_{k}\right) \cdot \mathrm{OD}_{i j 3} \cdot \frac{f_{\mathrm{B}}}{f_{\mathrm{A}}+f_{\mathrm{B}}}\right]\right\} .
\end{aligned}
$$

The total passenger in-vehicle time cost riding fleet $\mathrm{A}$ and fleet $\mathrm{B}$ in one direction, or $C_{v}$, is the sum of $C_{v}^{\mathrm{A}}+C_{v}^{\mathrm{B}}$.

As regards operator cost, the operator cost is a function of vehicle running time. The running time from the starting station to the ending station includes the running time between stations, as well as the dwell time and acceleration or deceleration delay. The operator cost of riding fleet $\mathrm{A}$ in one direction (named $C_{o}^{\mathrm{A}}$ ) can be written as follows:

$$
\begin{aligned}
C_{o}^{\mathrm{A}=} & O \cdot f_{\mathrm{A}} \\
& \cdot \sum_{k=1}^{N-1}\left(\frac{d_{k}}{v}+\max \left[a \cdot \lambda_{k}^{\mathrm{A}+}, b \cdot \lambda_{k}^{\mathrm{A}-}\right]+\frac{2 v}{g}\right),
\end{aligned}
$$

where $O$ is the running time value.

The operator cost of riding fleet $\mathrm{B}$ in one direction (named $C_{o}^{\mathrm{B}}$ ) can be shown as follows:

$$
\begin{aligned}
C_{o}^{\mathrm{B}}= & O \cdot f_{\mathrm{B}} \\
& \cdot \sum_{k=1}^{N-1}\left(\frac{d_{k}}{v}+\max \left[a \cdot \lambda_{k}^{\mathrm{B}+}, b \cdot \lambda_{k}^{\mathrm{B}-}\right]+\frac{2 v}{g} \cdot x_{k}\right) .
\end{aligned}
$$

The total operator cost in one direction, or $C_{o}$, is the sum of $C_{o}^{\mathrm{A}}+C_{o}^{\mathrm{B}}$.

The above models are used to determine one-direction user costs and operator costs. The other direction models are similar to those used for one direction. Thus, user cost and operator cost in the other directions (such as $C_{W}^{\prime}, C_{V}^{\prime}$, and $C_{o}^{\prime}$ ) can be determined. To ensure that the numbers of vehicles will be even at the end stations, the frequencies in both directions are equal in all-stop line or limited services.

Therefore, the objective function of the total cost in allstop line and limited services, denoted as $C$, is the sum of the variable cost items given above, which expresses the waiting time, in-vehicle time, and operator cost. It is formulated as follows:

$$
\begin{array}{ll}
\min & C=C_{W}+C_{V}+C_{o}+C_{W}^{\prime}+C_{V}^{\prime}+C_{o}^{\prime} \\
\text { s.t. } & q_{\max \mathrm{A}} \leq \eta \cdot K \\
& q_{\max \mathrm{B}} \leq \eta \cdot K,
\end{array}
$$

where $K$ is the vehicle capacity; $\eta$ is the vehicle occupancy rate, and $q_{\max \text { A }}$ and $q_{\max \text { B }}$ are the maximum loads of fleet $A$ and fleet $B$ in the sections between stations, which can be formulated as follows:

$$
\begin{aligned}
q_{\max \mathrm{A}}=\max \left[\sum_{k=1}^{l}\left(\lambda_{k}^{\mathrm{A}+}-\lambda_{k}^{\mathrm{A}-}\right)\right] & \\
& \forall l=1,2, \ldots, N-1,
\end{aligned}
$$

$$
\begin{aligned}
& q_{\max \mathrm{B}}=\max \left[\sum_{k=1}^{l}\left(\lambda_{k}^{\mathrm{B}+}-\lambda_{k}^{\mathrm{B}-}\right)\right], \\
& \forall l=1,2, \ldots, N-1 .
\end{aligned}
$$

3.2. Model for the Limited-Stop and Short-Turn Line. Thus far, the deficiency in studies related to limited-stop services is that those studies do not integrate short turning between different lines. By considering short turning, bus running times can be reduced by using shorter cycles. Such behavior is an integral feature of bus systems based on limited-stop services. The model that is based on limited-stop services adds the potential for short turning to the objective function that imposes shorter running times, due to the fact that buses will run without passengers at either end of the line. In this paper, we assume that the buses of fleet $\mathrm{B}$ run between stations $m+1$ and $N-n$. The shorter running time cost, $C_{s}$, can be calculated by the following model:

$$
C_{s}=O \cdot f_{\mathrm{B}} \cdot 2\left(\sum_{k=1}^{m} \frac{d_{k}}{v}+\sum_{k=N-n}^{N-1} \frac{d_{k}}{v}\right) .
$$

The variables in the model are $m$ and $n$, which are, respectively, the minimal numbers of consecutive skipped stations at either end of the line. Figure 4 shows the determination of the starting station and ending station of a short-turn service. Parameter $m$ belongs to the sets $X_{m_{1}}^{1}$ and $X_{m_{2}}^{2}$ in directions 1 and 2, while parameter $n$ belongs to sets $X_{n_{1}}^{1}$ and $X_{n_{2}}^{2}$.

Parameters $m$ and $n$ can be determined as follows:

$$
\begin{aligned}
& m=\min \left[\operatorname{size}\left(X_{m_{1}}^{1}\right), \operatorname{size}\left(X_{m_{2}}^{2}\right)\right], \\
& n=\min \left[\operatorname{size}\left(X_{n_{1}}^{1}\right), \operatorname{size}\left(X_{n_{2}}^{2}\right)\right], \\
& X_{m_{1}}^{1}=\left\{x_{m_{1}}^{1} \mid \prod_{i=1}^{m_{1}}\left(1-x_{i}^{1}\right)=0\right\}, \\
& X_{m_{2}}^{2}=\left\{x_{m_{2}}^{2} \mid \prod_{i=1}^{m_{2}}\left(1-x_{i}^{2}\right)=0\right\}, \quad \forall m_{1}=1,2,3, \ldots, \\
& X_{n_{1}}^{1}=\left\{x_{n_{1}}^{1} \mid \prod_{i=n_{1}}^{N}\left(1-x_{i}^{1}\right)=0\right\}, \\
& \forall m_{2}=1,2,3, \ldots,
\end{aligned}
$$




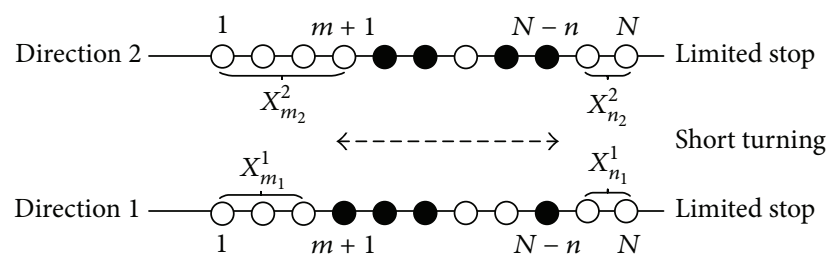

FIGURE 4: Determination of starting station and ending station of short turning.

$$
\begin{aligned}
X_{n_{2}}^{2}=\left\{x_{n_{2}}^{2} \mid \prod_{i=n_{2}}^{N}\left(1-x_{i}^{2}\right)\right. & =0\}, \\
& \forall n_{2}=N, N-1, N-2, \ldots,
\end{aligned}
$$

where the variables $x_{i}^{1}$ and $x_{i}^{2}$ indicate the type of station $i$ in direction 1 and direction 2 .

The objective function for determining the total cost of integrating the limited-stop and short-turning service, $C$, is the limited-stop cost minus the short-turn cost. This total cost is formulated as follows:

$$
\begin{array}{ll}
\min & C=C_{W}+C_{V}+C_{o}+C_{W}^{\prime}+C_{V}^{\prime}+C_{o}^{\prime}-C_{s} \\
\text { s.t. } & q_{\max \mathrm{A}} \leq \eta \cdot K \\
& q_{\max \mathrm{B}} \leq \eta \cdot K .
\end{array}
$$

\section{Solution Methods}

Now, we need a solution algorithm for the above models in order to find an operational scheme and yield satisfactory frequencies for fleet A and fleet B. A genetic algorithm could be used to yield a minimum-cost transit operation for optimizing transit routes and bus frequencies [22, 23]. The objective function is minimized by an optimization program coded in MATLAB R2011a.

For the genetic algorithm, each gene location in a chromosome which is randomly generated represents a possible type of station, where " 1 " indicates stops and " 0 " indicates skips at the corresponding station. A chromosome consists of entire schemes of bus routes, as shown as follows:

$$
\left[x_{1}, x_{2}, \ldots, x_{N}, x_{N+1}, x_{N+2}, \ldots, x_{2 N}\right]
$$

where $x_{1}, x_{2}, \ldots, x_{N}$ are the types of stations in direction 1 and $x_{N+1}, x_{N+2}, \ldots, x_{2 N}$ are the types of stations in direction 2 . Then, via selecting and reproducing, crossover, and mutation, the chromosome can be generated after several iterations.

As the buses run in both directions, the results of the model must provide the same frequencies with the same services in each direction. Therefore, $N$ stops are actually modeled as a one-way corridor with $2 N$ stops, with first $N$ representing one direction and last $N$ representing the other. An O/D matrix and set $d_{k}$ can be transformed, as shown in Figure 5. Afterwards, three types of services are considered,

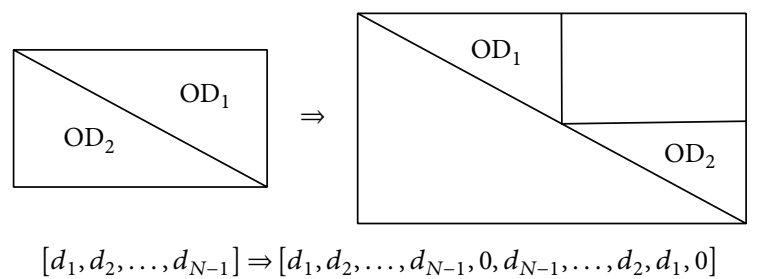

FIgURE 5: Transformation of the O/D matrix and set $d_{k}$.

TABLE 2: O/D matrix of Example 1.

\begin{tabular}{lcccccccccc}
\hline $\mathrm{O} \backslash \mathrm{D}$ & 1 & 2 & 3 & 4 & 5 & 6 & 7 & 8 & 9 & 10 \\
\hline 1 & 0 & 4 & 6 & 2 & 3 & 8 & 2 & 6 & 3 & 3 \\
2 & 4 & 0 & 6 & 6 & 5 & 6 & 2 & 7 & 3 & 2 \\
3 & 3 & 5 & 0 & 12 & 3 & 2 & 3 & 7 & 7 & 3 \\
4 & 6 & 2 & 4 & 0 & 18 & 45 & 13 & 58 & 6 & 3 \\
5 & 1 & 4 & 3 & 3 & 0 & 255 & 67 & 468 & 52 & 47 \\
6 & 4 & 7 & 9 & 1 & 17 & 0 & 82 & 479 & 154 & 57 \\
7 & 1 & 6 & 2 & 7 & 278 & 13 & 0 & 107 & 22 & 14 \\
8 & 3 & 4 & 4 & 3 & 355 & 14 & 92 & 0 & 39 & 21 \\
9 & 2 & 2 & 2 & 4 & 54 & 9 & 13 & 2 & 0 & 5 \\
10 & 3 & 2 & 3 & 2 & 16 & 3 & 4 & 2 & 2 & 0 \\
\hline
\end{tabular}

TABle 3: O/D matrix of Example 2.

\begin{tabular}{lcccccccccc}
\hline $\mathrm{O} \backslash \mathrm{D}$ & 1 & 2 & 3 & 4 & 5 & 6 & 7 & 8 & 9 & 10 \\
\hline 1 & 0 & 110 & 9 & 5 & 10 & 12 & 64 & 182 & 294 & 432 \\
2 & 300 & 0 & 1 & 2 & 1 & 2 & 1 & 4 & 6 & 6 \\
3 & 85 & 4 & 0 & 3 & 0 & 2 & 4 & 1 & 10 & 2 \\
4 & 148 & 8 & 5 & 0 & 1 & 2 & 2 & 4 & 2 & 4 \\
5 & 33 & 2 & 2 & 2 & 0 & 2 & 1 & 5 & 2 & 4 \\
6 & 19 & 11 & 9 & 3 & 2 & 0 & 4 & 0 & 6 & 4 \\
7 & 30 & 2 & 2 & 5 & 2 & 1 & 0 & 2 & 2 & 2 \\
8 & 243 & 15 & 12 & 13 & 11 & 8 & 9 & 0 & 98 & 84 \\
9 & 179 & 2 & 3 & 2 & 1 & 1 & 2 & 1 & 0 & 16 \\
10 & 482 & 24 & 25 & 23 & 21 & 16 & 20 & 22 & 18 & 0 \\
\hline
\end{tabular}

namely, (1) an all-stop-only service without any limitedstop or a short-turn service, (2) an all-stop and limited-stop service without short-turn service, and (3) an all-stop with limited-stop and short-turn service. We examine each service to search for the minimum cost.

\section{Numerical Applications}

Now, the methodology proposed in the above section is applied to a bus corridor comprising 10 stations. The $\mathrm{O} / \mathrm{D}$ matrices of our two examples are shown in Tables 2 and 3. In order to reflect the effect of an integrated limited-stop and short-turn strategy, numerical applications consider the three strategies: (i) an all-stop-only service (single frequency), which serves every stop; (ii) a limited-stop-only service, which serves a subset of stops; (iii) an integrated limited-stop and short-turn strategy, which serves a subset of stops in a shorter cycle. 
TABLE 4: Results of Example 1.

\begin{tabular}{|c|c|c|c|c|}
\hline Service & Operational scheme & $f_{\mathrm{A}}(\mathrm{veh} / \mathrm{h})$ & $f_{\mathrm{B}}(\mathrm{veh} / \mathrm{h})$ & $C(\$ / \mathrm{h})$ \\
\hline Only all stops & {$\left[\begin{array}{l}0000000000 \\
0000000000\end{array}\right]$} & 60 & - & 1235 \\
\hline Only limited stop & $\left.\begin{array}{l}0001110111 \\
0000101110\end{array}\right]$ & 41 & 21 & 1228 \\
\hline Integrated limited stop and short turning & 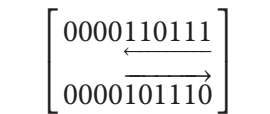 & 27 & 52 & 1171 \\
\hline
\end{tabular}

TABLE 5: Results of Example 2.

\begin{tabular}{lccr}
\hline Service & Operational scheme & $f_{\mathrm{A}}(\mathrm{veh} / \mathrm{h})$ & $f_{\mathrm{B}}(\mathrm{veh} / \mathrm{h})$ \\
\hline Only all stops & {$\left[\begin{array}{l}0000000000 \\
0000000000\end{array}\right]$} & 62 & - \\
& {$\left[\begin{array}{c}1000000111 \\
1110000001\end{array}\right]$} & 42 & 2128 \\
Only limited stop & - & - & 2109 \\
Integrated limited stop and short turning & - & - \\
\hline
\end{tabular}

According to our study data, the spacing between interfacing stops is $0.555 \mathrm{~km}$. The average bus speed is $26 \mathrm{~km} / \mathrm{h}$, and the accelerated speed is $0.77 \mathrm{~m} / \mathrm{s}^{2}$. The average passenger boarding and alighting times, respectively, are $3.8 \mathrm{~s}$ and $3 \mathrm{~s}$. Time value is assumed to be $4 \$ / \mathrm{pax} / \mathrm{h}$ for waiting time, $3 \$ / \mathrm{pax} / \mathrm{h}$ for in-vehicle time, and $10 \$ / \mathrm{veh}-\mathrm{h}$ for running time. The vehicle capacity is $80 \mathrm{pax} / \mathrm{veh}$, and the vehicle occupancy rate is 0.9 .

The results of Example 1, as shown in Table 4, indicate that only limited-stop services and integrated limited-stop and short-turn services can be implemented to Example 1, only if it is feasible for fleet B to turn back at station 5 without physical or operational constraints and if the two services can reduce the total cost of the transit system. Under the limitedstop-only service, fleet B buses skip stations 1, 2, 3, 4, 6, and 10 in direction 1 . In direction 2 , stations $1,2,3$, and 7 are skipped. The buses operate the entire route. Compared with a limitedstop-only service, after the optimization calculations, station 4 is skipped in direction 2 in the integrated strategy. This change could save a large amount of vehicle running time, despite increasing user costs. Meanwhile, the frequencies of buses have been changed, and the frequency of fleet B has been increased. The integrated strategy has a better effect than the limited-stop-only service. The total cost is changed from $1235 \$ / \mathrm{h}$ to $1171 \$ / \mathrm{h}$. Under our integrated strategy, the buses of fleet B skip stations $1,2,3,4,6$, and 10 in direction 1 . In direction 2, stations 1, 2, 3, 4, and 7 are skipped. The buses operate between stations 5 and 10 , which greatly reduces running time. Unlike Example 1, Table 5 shows the results of Example 2. Here, the integrated limited-stop and shortturn service cannot be used, because there is no station where buses run without passengers. Under a limited-stoponly service, fleet B buses skip a subset of stations in order to reduce in-vehicle time for passengers and running time for vehicles.

A sensitivity analysis of the objective function value with weighting user and operator costs for Example 1 is shown in Figure 6. An adjustment of vehicle running time value means operator costs are higher or lower than the base data. The sensitivity analysis also reveals that, with the different values of vehicle running times, the integrated strategy can greatly reduce total costs. Although the limitedstop-only service also has an effect in terms of improving the benefits of a transit system, the limited-stop-only service is not as good as our integrated strategy. Meanwhile, Table 6 presents the optimized frequencies with the different values of vehicle running times. By increasing the running time value, the optimized frequencies are decreased, because of high operator costs.

\section{Conclusions}

In this paper, we have presented a model for designing an integrated limited-stop and short-turn service to offer the optimal frequencies of buses. Our strategy could minimize the total cost of a transit system, including user costs and operator costs. Using a trip matrix, the model can be solved. Since our integrated service is different from the traditional service (single frequency), we need to determine the appropriate skipped stations and turning back stations in a line, and the appropriate stations can be obtained from the model. By skipping some stations with low demand, the integrated strategy can offer higher frequency in those segments with high demand. To test the model, two numerical applications were conducted, which reflect the effects of three services (allstop-only, limited-stop-only, and integrated limited-stop and 
TABLE 6: Optimized frequencies versus operator's value of running time.

\begin{tabular}{|c|c|c|c|c|c|}
\hline \multirow{2}{*}{ Vehicle running time value ( $\$ / v e h-h)$} & \multicolumn{2}{|c|}{ Integrated strategy } & \multicolumn{2}{|c|}{ Limited stop } & \multirow{2}{*}{$\begin{array}{c}\text { All stops } \\
f_{\mathrm{A}}(\mathrm{veh} / \mathrm{h})\end{array}$} \\
\hline & $f_{\mathrm{A}}(\mathrm{veh} / \mathrm{h})$ & $f_{\mathrm{B}}(\mathrm{veh} / \mathrm{h})$ & $f_{\mathrm{A}}(\mathrm{veh} / \mathrm{h})$ & $f_{\mathrm{B}}(\mathrm{veh} / \mathrm{h})$ & \\
\hline 10 & 27 & 52 & 41 & 21 & 60 \\
\hline 12.5 & 25 & 45 & 38 & 18 & 54 \\
\hline 15 & 23 & 41 & 35 & 16 & 49 \\
\hline 17.5 & 21 & 38 & 33 & 14 & 45 \\
\hline 20 & 20 & 35 & 31 & 13 & 42 \\
\hline
\end{tabular}

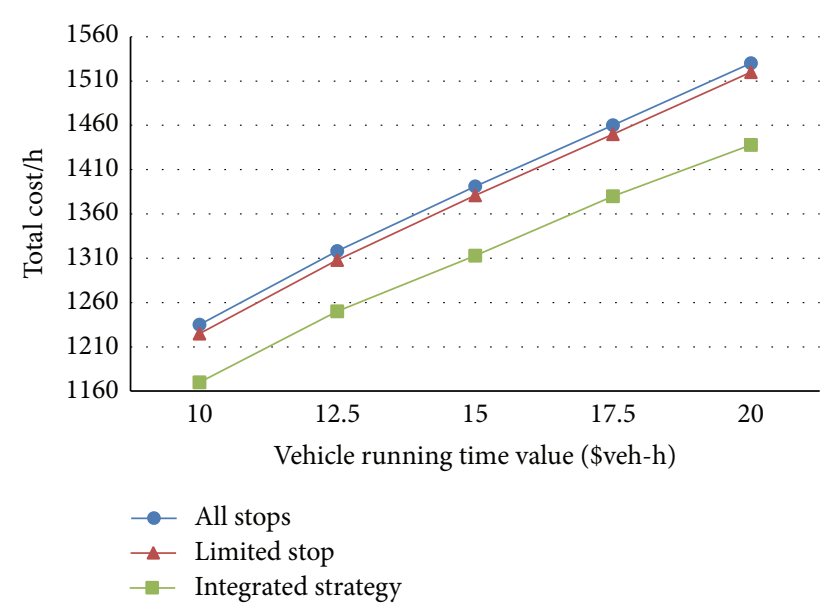

FIGURE 6: Total costs versus operator's value of running time.

short-turn services). The significant conclusions of this work can be found.

When the model is applied to a trip matrix, two possible optimal services can be proposed which take into account the form of demand on the entire line. Those two possible services are the limited-stop-only service and an integrated limited-stop and short-turn service. If demand is high at both ends of the line, the short-turn service cannot be implemented, because there is no suitable station from which to turn back.

The integrated limited-stop and short-turn service which results from our model has a better effect on reducing total costs compared to the all-stop-only and limited-stop-only services.

By weighting user and operator costs, our integrated limited-stop service can accrue relative advantages over a single-frequency service. In addition, with high operator costs, the optimal frequencies are decreasing, which in turn will reduce total costs.

\section{Competing Interests}

The authors declare that they have no competing interests.

\section{Acknowledgments}

The work was supported by the National Natural Science Foundation of China (Grant no. 51378237).

\section{References}

[1] P. G. Furth and F. B. Day, "Transit routing and scheduling strategies for heavy demand corridors," Transportation Research Record, vol. 1011, pp. 23-26, 1985.

[2] V. Chiraphadhanakul and C. Barnhart, "Incremental bus service design: combining limited-stop and local bus services," Public Transport, vol. 5, no. 1-2, pp. 53-78, 2013.

[3] O. J. Ibarra-Rojas, F. Delgado, R. Giesen, and J. C. Muñoz, "Planning, operation, and control of bus transport systems: a literature review," Transportation Research Part B: Methodological, vol. 77, pp. 38-75, 2015.

[4] H. Larrain, J. C. Muñoz, and R. Giesen, "Generation and design heuristics for zonal express services," Transportation Research Part E: Logistics \& Transportation Review, vol. 79, pp. 201-212, 2015.

[5] Y. Y. Ulusoy and S. I.-J. Chien, "Optimal bus service patterns and frequencies considering transfer demand elasticity with genetic algorithm," Transportation Planning and Technology, vol. 38, no. 4, pp. 409-424, 2015.

[6] W. C. Jordan and M. A. Turnquist, "Zone scheduling of bus routes to improve service reliability," Transportation Science, vol. 13, no. 3, pp. 242-268, 1979.

[7] P. Furth, "Short turning on transit routes," Transportation Research Record, vol. 1108, pp. 42-52, 1987.

[8] A. Ceder, "Optimal design of transit short-turn trips," Transportation Research Record, vol. 1221, p. 22, 1989.

[9] P. Delle Site and F. Filippi, "Service optimization for bus corridors with short-turn strategies and variable vehicle size," Transportation Research Part A: Policy and Practice, vol. 32, no. 1, pp. 19-38, 1998.

[10] P. G. Furth, "Alternating deadheading in bus route operations," Transportation Science, vol. 19, no. 1, pp. 13-28, 1985.

[11] X. J. Eberlein, N. H. M. Wilson, C. Barnhart, and D. Bernstein, "The real-time deadheading problem in transit operations control," Transportation Research, Part B: Methodological, vol. 32, no. 2, pp. 77-100, 1997.

[12] Z. Liu, Y. Yan, X. Qu, and Y. Zhang, "Bus stop-skipping scheme with random travel time," Transportation Research Part C: Emerging Technologies, vol. 35, pp. 46-56, 2013.

[13] L. L. Afanasiev and S. Y. Liberman, "Principles for organizing express bus services," Transportation Research Part A: General, vol. 17, no. 5, pp. 343-346, 1983.

[14] N. C. Silverman, "Limited-stop bus service at New York city transit," Journal of Transportation Engineering, vol. 124, no. 6, pp. 503-509, 1998.

[15] P. R. Tétreault and A. M. El-Geneidy, "Estimating bus run times for new limited-stop service using archived AVL and APC data," Transportation Research Part A: Policy and Practice, vol. 44, no. 6, pp. 390-402, 2010. 
[16] C. Leiva, J. C. Muñoz, R. Giesen, and H. Larrain, "Design of limited-stop services for an urban bus corridor with capacity constraints," Transportation Research Part B: Methodological, vol. 44, no. 10, pp. 1186-1201, 2010.

[17] S. Feng, Z. Wen-tao, Y. Ying, and W. Dian-hai, "Optimal skipstop schedule under mixed traffic conditions for minimizing travel time of passengers," Discrete Dynamics in Nature and Society, vol. 2013, Article ID 930562, 8 pages, 2013.

[18] X. Chen, B. Hellinga, C. Chang, and L. Fu, "Optimization of headways with stop-skipping control: a case study of bus rapid transit system," Journal of Advanced Transportation, vol. 49, no. 3, pp. 385-401, 2015.

[19] H. Niu, X. Zhou, and R. Gao, “Train scheduling for minimizing passenger waiting time with time-dependent demand and skipstop patterns: nonlinear integer programming models with linear constraints," Transportation Research Part B: Methodological, vol. 76, pp. 117-135, 2015.

[20] J. Chen, Z. Liu, S. Zhu, and W. Wang, "Design of limited-stop bus service with capacity constraint and stochastic travel time," Transportation Research Part E: Logistics and Transportation Review, vol. 83, pp. 1-15, 2015.

[21] C. E. Cortés, S. Jara-Díaz, and A. Tirachini, "Integrating short turning and deadheading in the optimization of transit services," Transportation Research Part A: Policy and Practice, vol. 45, no. 5, pp. 419-434, 2011.

[22] J. Agrawal and T. V. Mathew, "Transit route network design using parallel genetic algorithm," Journal of Computing in Civil Engineering, vol. 18, no. 3, pp. 248-256, 2004.

[23] B. Yu, Z. Yang, and J. Yao, "Genetic algorithm for bus frequency optimization," Journal of Transportation Engineering, vol. 136, no. 6, Article ID 002006QTE, pp. 576-583, 2010. 


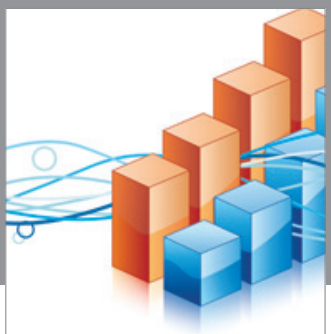

Advances in

Operations Research

vatem alat4

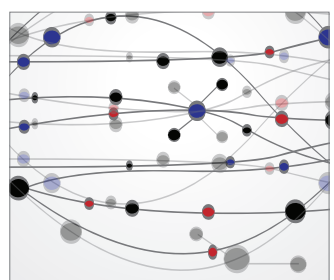

\section{The Scientific} World Journal
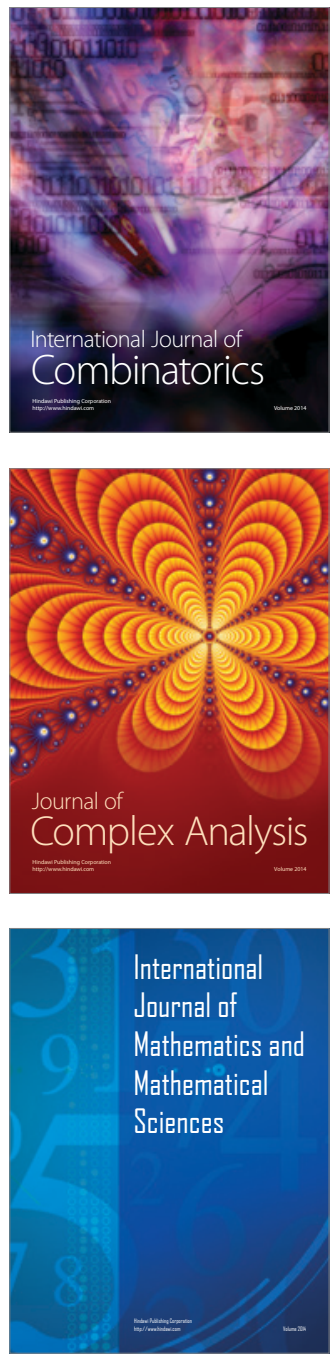
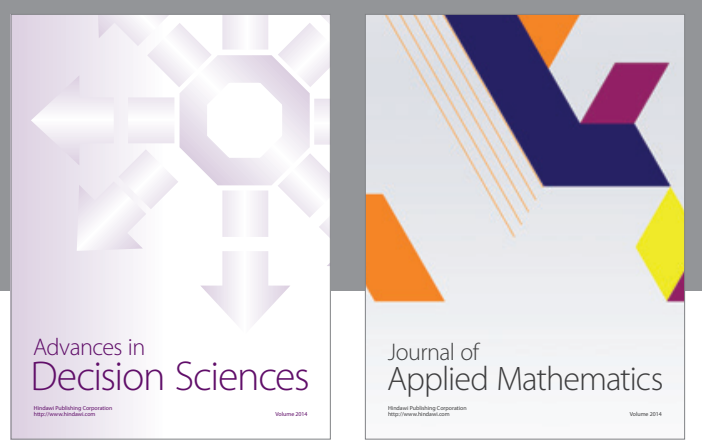

Algebra

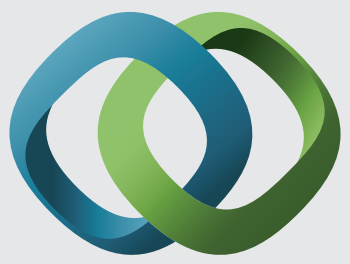

\section{Hindawi}

Submit your manuscripts at

http://www.hindawi.com
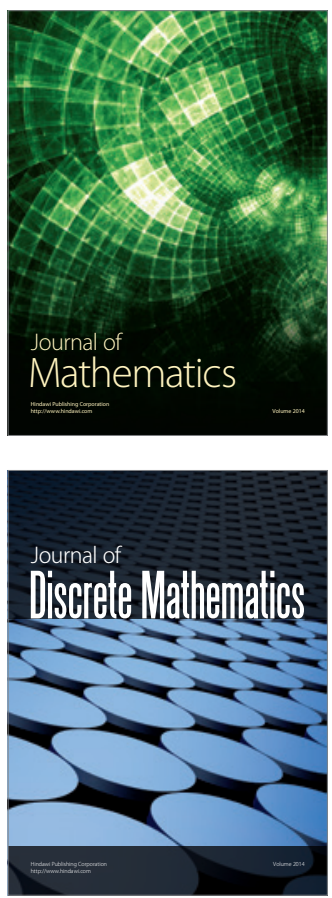

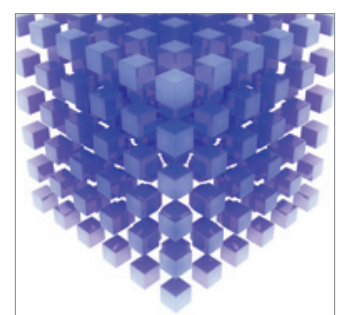

Mathematical Problems in Engineering
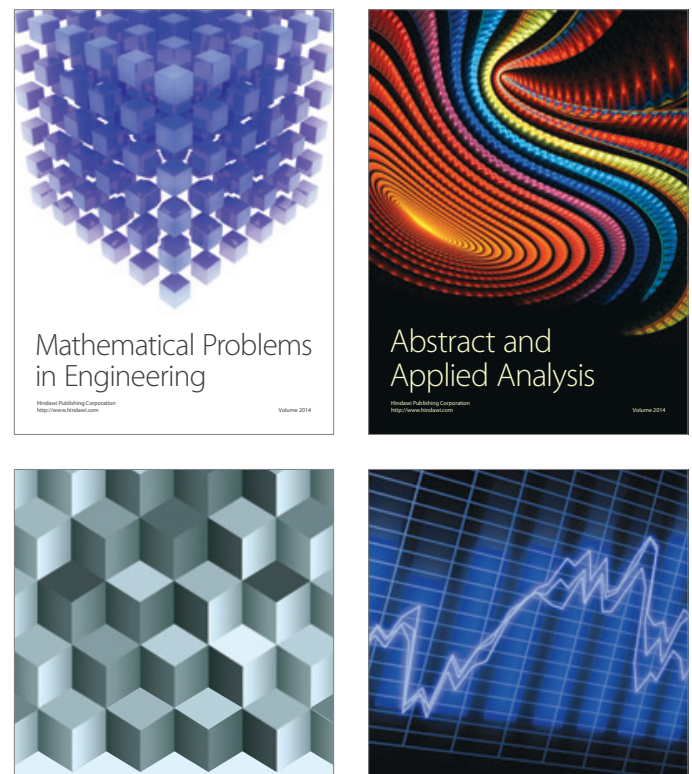

Journal of

Function Spaces

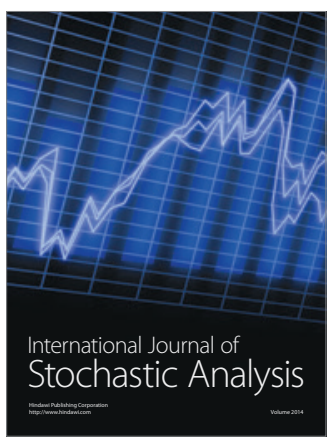

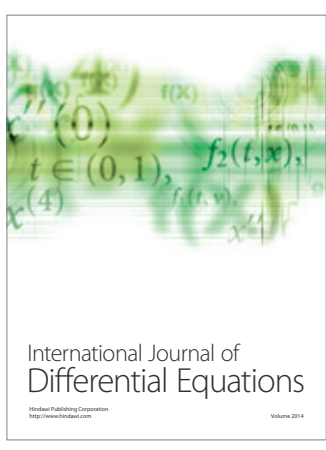
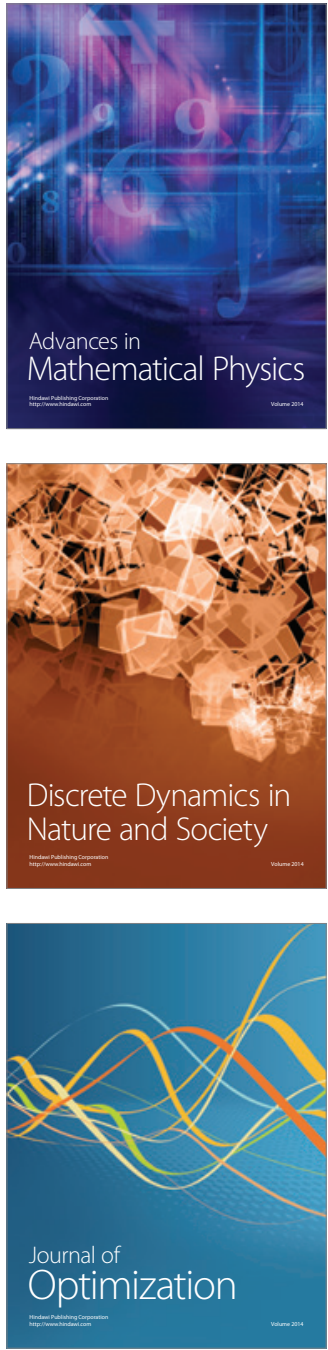